University of Wollongong

Research Online

Australian Institute for Innovative Materials -

Papers

Australian Institute for Innovative Materials

$1-1-2013$

\title{
Enhanced pinning in superconducting thin films with graded pinning landscapes
}

M Motta

Federal University Of Sao Carlos, Brazil

F Colauto

Federal University Of Sao Carlos, Brazil

W A. Ortiz

Federal University Of Sao Carlos, Brazil

J Fritzsche

Chalmers University Of Technology, Sweden

J Cuppens

Catholic University of Leuven

See next page for additional authors

Follow this and additional works at: https://ro.uow.edu.au/aiimpapers

Part of the Engineering Commons, and the Physical Sciences and Mathematics Commons

Research Online is the open access institutional repository for the University of Wollongong. For further information contact the UOW Library: research-pubs@uow.edu.au 


\title{
Enhanced pinning in superconducting thin films with graded pinning landscapes
}

\author{
Abstract \\ A graded distribution of antidots in superconducting a-Mo $79 \mathrm{Ge} 21$ thin films has been investigated by \\ magnetization and magneto-optical imaging measurements. The pinning landscape has maximum \\ density at the sample border, decreasing linearly towards the center. Its overall performance is noticeably \\ superior than that for a sample with uniformly distributed antidots: For high temperatures and low fields, \\ the critical current is enhanced, whereas the region of thermomagnetic instabilities in the field- \\ temperature diagram is significantly suppressed. These findings confirm the relevance of graded \\ landscapes on the enhancement of pinning efficiency, as recently predicted by Misko and Nori [Phys. Rev. \\ B 85, 184506 (2012)]. 2013 AIP Publishing LLC.

\section{Keywords} \\ pinning, enhanced, graded, films, thin, landscapes, superconducting \\ Disciplines \\ Engineering | Physical Sciences and Mathematics

\section{Publication Details} \\ Motta, M., Colauto, F., Ortiz, W. A., Fritzsche, J., Cuppens, J., Gillijns, W., Moshchalkov, V. V., Johansen, T. \\ H., Sanchez, A. \& Silhanek, A. (2013). Enhanced pinning in superconducting thin films with graded pinning \\ landscapes. Applied Physics Letters, 102 (21), 212601-1-212601-5.

\section{Authors} \\ M Motta, F Colauto, W A. Ortiz, J Fritzsche, J Cuppens, W Gillijns, V V. Moshchalkov, T H. Johansen, A W \\ Sanchez, and A Silhanek
}




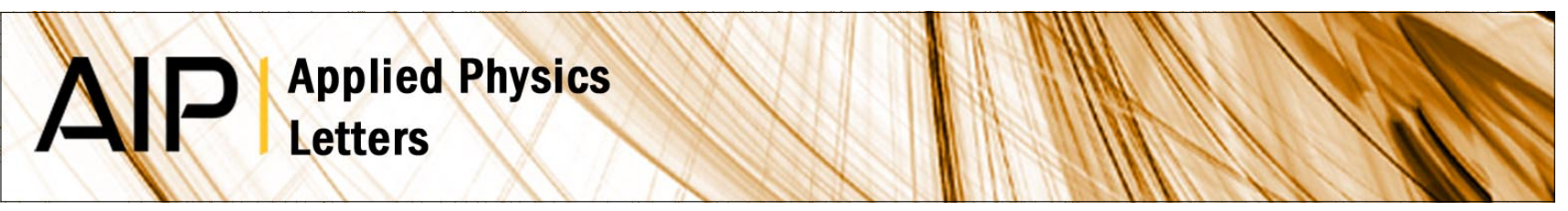

\section{Enhanced pinning in superconducting thin films with graded pinning landscapes}

M. Motta, F. Colauto, W. A. Ortiz, J. Fritzsche, J. Cuppens et al.

Citation: Appl. Phys. Lett. 102, 212601 (2013); doi: 10.1063/1.4807848

View online: http://dx.doi.org/10.1063/1.4807848

View Table of Contents: http://apl.aip.org/resource/1/APPLAB/v102/i21

Published by the AIP Publishing LLC.

Additional information on Appl. Phys. Lett.

Journal Homepage: http://apl.aip.org/

Journal Information: http://apl.aip.org/about/about_the_journal

Top downloads: http://apl.aip.org/features/most_downloaded

Information for Authors: http://apl.aip.org/authors

\section{ADVERTISEMENT}
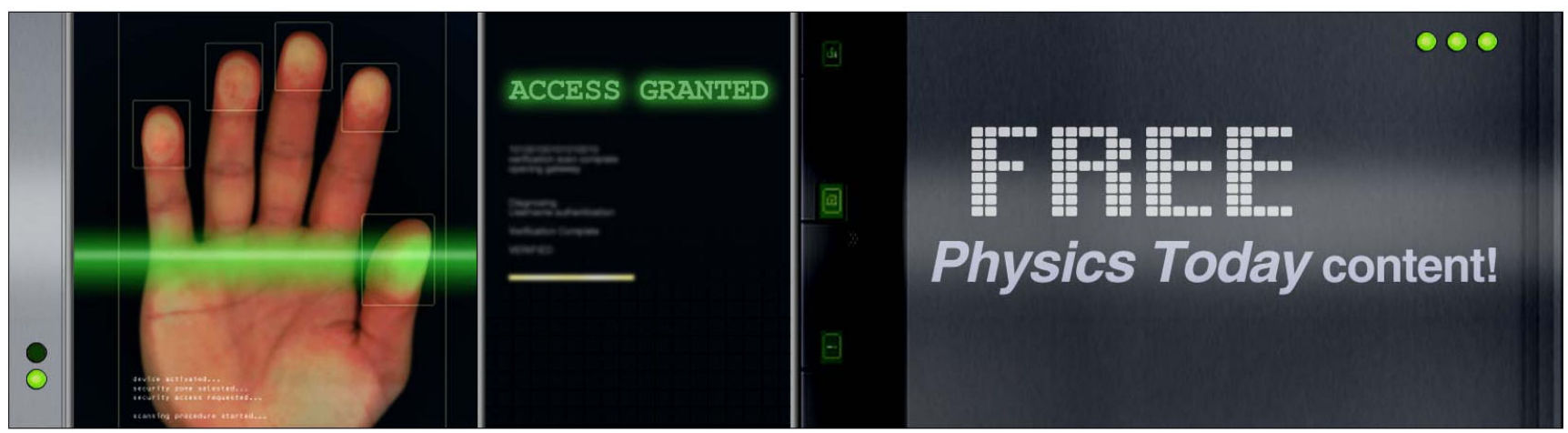


\title{
Enhanced pinning in superconducting thin films with graded pinning landscapes
}

\author{
M. Motta, ${ }^{1}$ F. Colauto, ${ }^{1}$ W. A. Ortiz, ${ }^{1}$ J. Fritzsche, ${ }^{2}$ J. Cuppens, ${ }^{3}$ W. Gillijns, ${ }^{3}$ \\ V. V. Moshchalkov, ${ }^{3}$ T. H. Johansen, ${ }^{4,5}$ A. Sanchez, ${ }^{6}$ and A. V. Silhanek ${ }^{7}$ \\ ${ }^{1}$ Departamento de Física, Universidade Federal de São Carlos, 13565-905 São Carlos, SP, Brazil \\ ${ }^{2}$ Department of Applied Physics, Chalmers University of Technology, S-412 96 Göteborg, Sweden \\ ${ }^{3}$ INPAC - Institute for Nanoscale Physics and Chemistry, Nanoscale Superconductivity and Magnetism Group, \\ K.U.Leuven, Celestijnenlaan 200D, B-3001 Leuven, Belgium \\ ${ }^{4}$ Institute for Superconducting and Electronic Materials, University of Wollongong, Northfields Avenue, \\ Wollongong, NSW 2522, Australia \\ ${ }^{5}$ Department of Physics, University of Oslo, P.O. Box 1048, Blindern, 0316 Oslo, Norway \\ ${ }^{6}$ Departament de Física, Universitat Autònoma de Barcelona, 08193 Bellaterra, Barcelona, Spain \\ ${ }^{7}$ Département de Physique, Université de Liège, B-4000 Sart Tilman, Belgium
}

(Received 13 November 2012; accepted 14 May 2013; published online 30 May 2013)

\begin{abstract}
A graded distribution of antidots in superconducting a- $\mathrm{Mo}_{79} \mathrm{Ge}_{21}$ thin films has been investigated by magnetization and magneto-optical imaging measurements. The pinning landscape has maximum density at the sample border, decreasing linearly towards the center. Its overall performance is noticeably superior than that for a sample with uniformly distributed antidots: For high temperatures and low fields, the critical current is enhanced, whereas the region of thermomagnetic instabilities in the field-temperature diagram is significantly suppressed. These findings confirm the relevance of graded landscapes on the enhancement of pinning efficiency, as recently predicted by Misko and Nori [Phys. Rev. B 85, 184506 (2012)]. @ 2013 AIP Publishing LLC. [http://dx.doi.org/10.1063/1.4807848]
\end{abstract}

In the mixed state of type-II superconductors, magnetic flux is admitted into the sample in the form of quantized vortices. When superconducting currents are present, vortices undergo a viscous motion, generating a local temperature rise. This unwanted consequence of vortex motion should be prevented, in order to avoid the weakening of the superconducting properties of the material, what would constitute a threat to its potential use in real applications. For this reason, the task of understanding vortex dynamics in the presence of pinning centers (PCs) has maintained, throughout the years, its status of a timely and relevant research problem for fundamental science and applications. ${ }^{1-4}$ A natural strategy in this attempt to anchor vortices is to spread, at random, small clusters of normal material, an approach adopted since long for superconducting alloys ${ }^{1}$ and reproduced more recently for high-temperature superconductors. ${ }^{2,4}$ Other commonly employed methods are the placement of arrays of magnetic dots on the surface of superconducting films, 5 and the creation of periodic arrays of antidots (ADs) in films. ${ }^{6}$

It has been demonstrated that the insertion of arrays of ADs in a superconducting film can lead, at high temperatures, to an increase of the critical current. ${ }^{6}$ Unfortunately, at low temperatures, such PCs facilitate the proliferation of flux channeling ${ }^{7-10}$ leading to unwanted instabilities of thermomagnetic origin ${ }^{11,12}$ which render the superconductor impractical. A good compromise between strong pinning and lack of channeling can be achieved by introducing a quasiperiodic array of PCs, as suggested theoretically by Misko et al. ${ }^{13-15}$ and confirmed experimentally by Kemmler et al. ${ }^{16}$ and Silhanek et al. ${ }^{17}$ This aperiodic distribution of pinning centers may be further optimized by matching it to the typically non-uniform distribution of vortices. For instance, in order to create a distribution of PCs compatible with zero-field cooling conditions, one should then distribute them with a density gradient, decreasing from the edges toward the center of the sample, as expected for the vortex distribution of a partially penetrated sample in the mixed state according to the Bean model. ${ }^{18-20}$ In agreement with this concept, Misko and Nori ${ }^{21}$ have recently predicted that an incommensurate array of PCs placed on the vertices of hyperbolic tessellations (i.e., a graded distribution of PCs) improves the pinning properties of superconductors. As a matter of fact, one major issue regarding the efficiency of vortex anchorage is to adapt the PC landscape in order to match the actual distribution of vortices.

In this letter, we demonstrate that an array of ADs creating a pinning landscape with PC density decreasing linearly from the edges toward the center of the thin film (the gradient sample) promotes an increase of the critical current. This enhancement is even better than that obtained when a homogeneous array of ADs (the uniform sample) is used. This array of PCs mimics approximately the flux profile described by the Bean model at high temperatures and low fields. Our results provide experimental confirmation of the recent theoretical predictions by Misko and Nori, ${ }^{21}$ although the graded pinning landscape employed here is somewhat less complex than the one proposed by them. Furthermore, although flux avalanches on the gradient sample are induced-as expected - by the presence of the array of pinning sites, this effect is comparatively less important than for the uniform sample. This is evidenced by the substantially smaller thermomagnetic instability (TMI) region on the magnetic fieldtemperature diagram for the gradient sample compared to the corresponding region for the uniform sample. Thus, the presence of a grid of ADs with density gradient not only generates the desired increase in critical current but also 
promotes an additional protection against the early occurrence of flux avalanches, as compared to the case of a film with a uniform distribution of ADs.

The samples investigated consist of amorphous $\mathrm{Mo}_{79} \mathrm{Ge}_{21}\left(\mathrm{a}-\mathrm{Mo}_{79} \mathrm{Ge}_{21}\right)$ thin films with thickness of $25 \mathrm{~nm}$, deposited by pulsed laser deposition on top of a $\mathrm{Si} / \mathrm{SiO}_{2}$ substrate. The pinning centers on both the gradient and the uniform sample consist of square holes of $0.5 \mu \mathrm{m}$ side, prepared by standard electron beam lithography. The lattice parameter of the uniform film (UNI) is $1 \mu \mathrm{m}$ and the lattice symmetry is square. At the edges of the gradient sample (GRAD) the separation between the centers of neighboring holes is also $1 \mu \mathrm{m}$, and its density varies inwards (but not laterally) with a constant increase on the row separation of $10 \mathrm{~nm} / \mathrm{row}$. Figures 1(a) and 1(b) left panel sketch the scheme of the spatial dependence of the density of ADs on the three films studied here. The right panels of Figure 1(b) show a zoomed up view obtained via atomic force microscopy of the edges and the center of sample GRAD, illustrating its density gradient. A third film (Plain), without ADs, was used as a reference sample.

The samples have a lithographically defined square shape with lateral dimensions of $1 \times 1 \mathrm{~mm}^{2}$ and the critical temperatures, $T_{c}$, are $6.73 \mathrm{~K}, 6.65 \mathrm{~K}$ and $7.10 \mathrm{~K}$, for samples Plain, UNI and GRAD, respectively. Using the temperature derivative of the upper critical field, the normal state resistivity and the dirty limit expressions, ${ }^{22}$ the zero-temperature superconducting coherence length $\left(\xi_{G L}(0)\right)$ and penetration depth $\left(\lambda_{G L}(0)\right)$ were estimated to be $5 \mathrm{~nm}$ and $500 \mathrm{~nm}$, respectively. Dc magnetization measurements were carried out in a commercial Quantum Design MPMS instrument. The magneto-optical technique employed for imaging the flux penetration morphology relies on the occurrence of the Faraday effect ${ }^{23}$ in an indicator film placed on top of the superconducting specimen. The indicators used in the

(a)

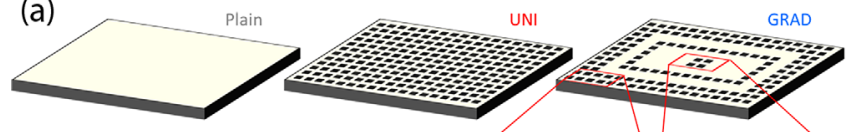

(b)
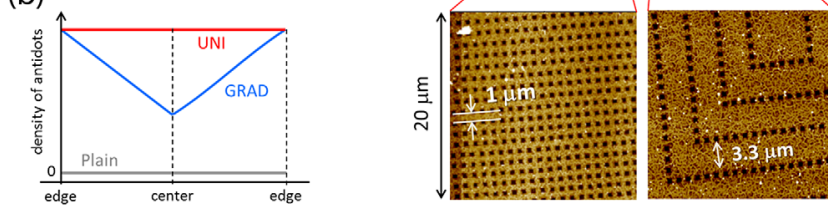
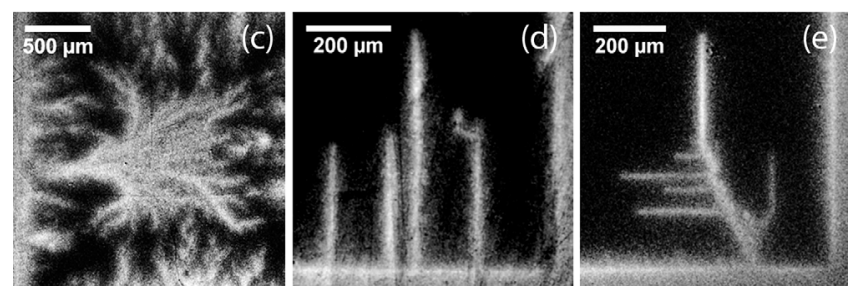

FIG. 1. (a) Scheme of the density of ADs for samples Plain, UNI, and GRAD (not to scale); (b) left panel: average areal density of antidots as a function of position for the three cases of pinning distribution considered in this work; (b) right panels: atomic force microscopy image showing the distribution of antidots at the border and at the center of the GRAD sample; (c) magneto-optical image, taken at $T=2.8 \mathrm{~K}$ and $H=2.2 \mathrm{Oe}$, for a sister plain sample (see Ref. 26); (d) same as (c) for sample UNI, at $T=4 \mathrm{~K}$ and $H=1.2 \mathrm{Oe}$; (e) sample GRAD at $T=4.75 \mathrm{~K}$ and $H=1.3 \mathrm{Oe}$. present work are Bi-substituted yttrium iron garnet films (Bi:YIG) with in-plane magnetization.

As already mentioned, within a limited interval of values of the applied field and temperature, flux avalanches originating from thermomagnetic instabilities are likely to develop in thin films of a variety of superconducting materials, ${ }^{24}$ including patterned a-Mo $\mathrm{Mo}_{79} \mathrm{Ge}_{21}$ thin films. ${ }^{25}$ However, this kind of catastrophic event in amorphous plain films of $\mathrm{Mo}_{79} \mathrm{Ge}_{21}$ was not reported so far. The lower panels of Figure 1 show representative examples of such avalanches in three a-Mo $\mathrm{Mo}_{79} \mathrm{Ge}_{21}$ films. Panel 1(c) shows dendritic structures, typical of plain films, here shown for a $2.5 \times 2.5 \mathrm{~mm}^{2}$ sample. ${ }^{26}$ Panels 1(d) and 1(e) exhibit straight tracks in the flux penetration, evidently guided by the rows of $\mathrm{ADs},{ }^{7,9,10}$ in the specimens UNI and GRAD, respectively.

Figure 2 depicts the effects on the critical current of a film of a- $\mathrm{Mo}_{79} \mathrm{Ge}_{21}$, caused by the insertion of different arrays of ADs. Panel (a) comprises magnetization loops for the three samples studied, taken at the reduced temperature $t=T / T_{c}=0.75$. At such temperatures, the loops for the samples with ADs are clearly wider and taller than that for the Plain film, confirming the enhanced pinning capability of the patterned samples, which implies larger critical currents for those specimens. It is particularly interesting to notice that, at large enough magnetic fields, sample UNI performs somewhat better than sample GRAD due to the fact that sample UNI has more ADs than sample GRAD, so that its

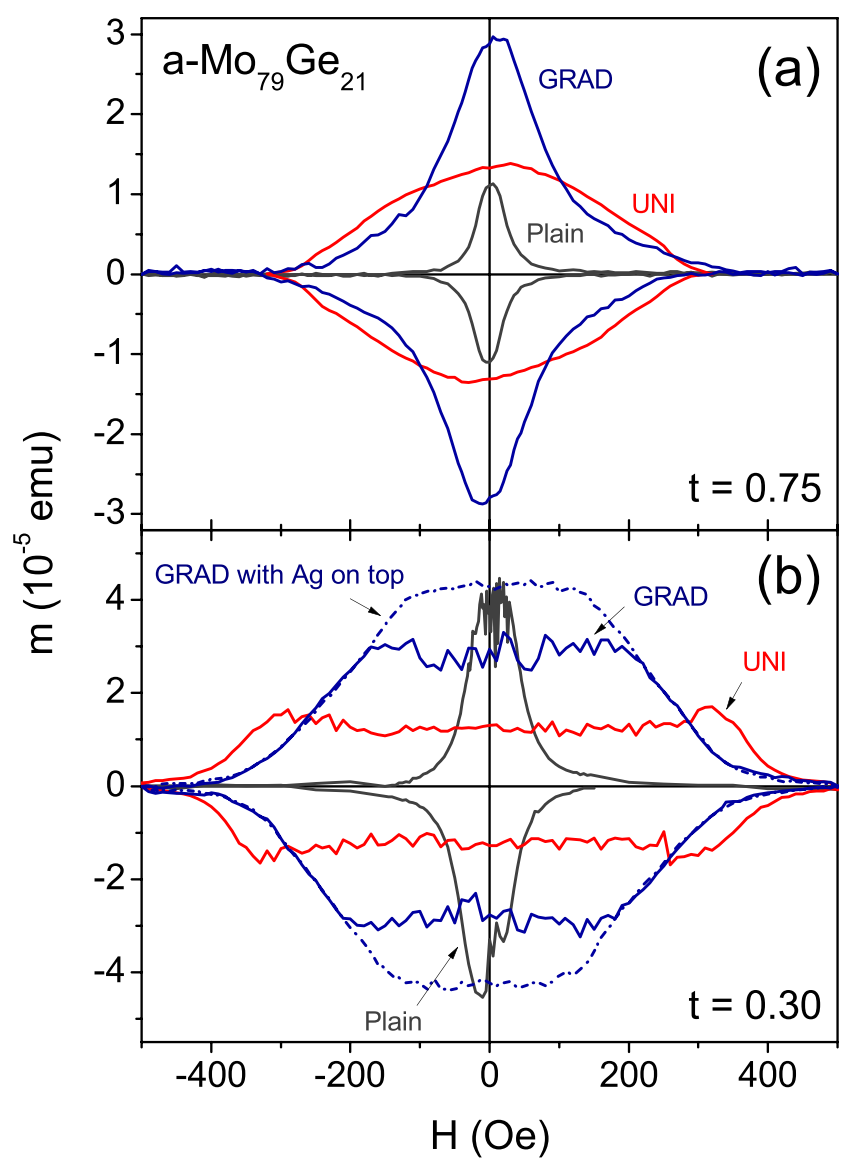

FIG. 2. (a) Dc magnetization versus magnetic field taken at the reduced temperature $t=0.75$ for samples Plain, UNI, and GRAD. (b) Magnetization loops for the 3 samples in the avalanche region $(t=0.30)$; a fourth loop is also shown for sample GRAD covered with a thick disk of Ag, an artifact employed to substantially suppress flux avalanches. 
pinning capability is better at larger fields. At fields below $60 \mathrm{Oe}$, however, the magnetic response of sample GRAD represents an enormous enhancement of the critical current, as compared with the uniform sample. At fields around zero, its increased pinning capability leads roughly to a factor of 2 on the critical current. We have obtained similar results in $\mathrm{Pb}$ samples with much smaller $\lambda / \xi$ ratio (not shown). The fact that no much difference is seen in the magnetization loop for increasing and decreasing field indicates that the graded distribution is equally efficient as a vortex dam preventing vortex entrance and exit and leading to a huge flux trapping at zero field.

The lower panel (b) in Figure 2 shows a similar set of hysteresis loops at the reduced temperature $t=T / T_{c}=0.30$. The noisy response at the central portion of the loops is the typical signature ${ }^{24,27}$ of the flux avalanches mentioned above. Alternative means to suppress such flux bursts have been already discussed in the literature, ${ }^{28,29}$ the simplest of which we have applied here. By placing a metallic disk (Ag) on top of sample GRAD, one manages to inhibit, via magnetic breaking, ${ }^{29}$ the thermomagnetic instabilities that trigger avalanches. The dotted-dashed curve in Figure 2(b) shows the hysteresis loop of sample GRAD with the Ag disk, indicating that flux avalanches are mostly suppressed in this configuration. One can thus conclude that the strategy of patterning a superconducting film with a gradient distribution of ADs is clearly more efficient in increasing the critical current than using a uniformly distributed array of ADs.

By repeating experiments as those depicted in Figure 2 for different values of the temperature, one can construct a magnetic field-temperature diagram containing the boundaries of the instability region for the samples studied. ${ }^{30}$ These frontiers are shown in Figure 3, from which one can clearly see that the inclusion of ADs enlarges the instability region, as compared to the Plain film. The region for sample GRAD is, however, substantially smaller than for sample UNI (notice the logarithmic scale on the lower portion of the vertical axis). Since these avalanches can be efficiently suppressed by depositing a metallic layer on top of the film of interest, one can fully appreciate the reach of the present results, from which we conclude that the strategy of inserting

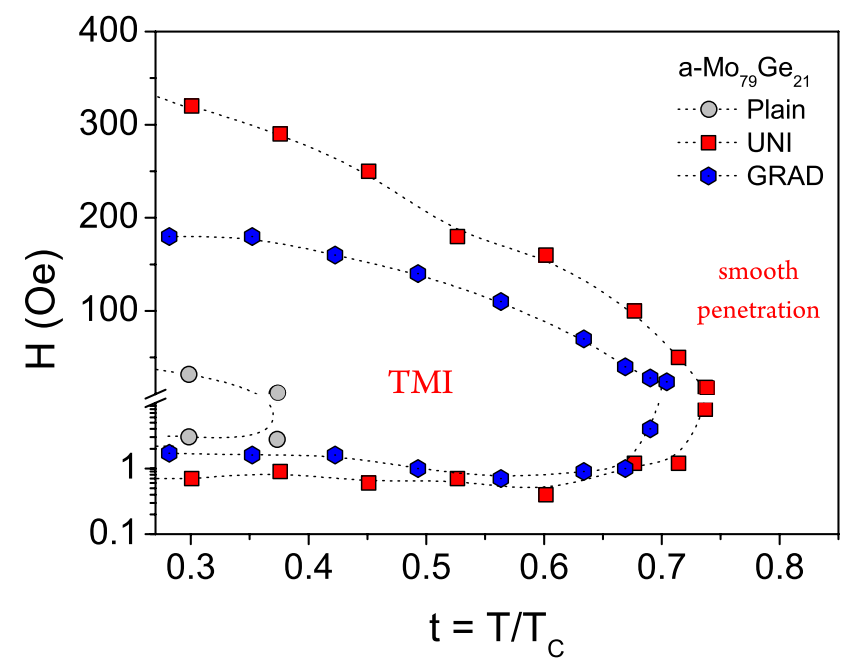

FIG. 3. Boundaries of the instability region of the studied a-Mo $\mathrm{Mo}_{79} \mathrm{Ge}_{21}$ thin films. Notice the logarithmic scale on the lower portion of the vertical axis. a graded pinning landscape represents an increased protection of the film against flux avalanches, a substantial advantage in terms of practical use of superconducting films in the presence of perpendicular magnetic fields. At the moment, it remains unclear whether this improved performance-better pinning at low fields and narrower TMI area in Figure 3-of the sample GRAD arises from the lack of perfect periodicity in the antidot lattice or from the gradient itself. In fact, this subject remains timely and relevant for the present efforts to enhance the critical current density of superconducting materials. ${ }^{13-15,31}$

In summary, we have demonstrated experimentally that, for low fields, a graded pinning landscape introduced in a superconducting film of a-Mo $\mathrm{Mo}_{79} \mathrm{Ge}_{21}$ increases the critical current, as compared to a uniform distribution of ADs. In addition, flux avalanche activity, typically induced by the presence of arrays of ADs, is less prejudicial for the sample with gradient distribution of antidots than for the uniformly distributed pinning centers. This work focuses on a particular gradient geometry following a very smooth linear decrease of areal density of pinning centers from the border of the sample towards its center. Further investigations in other gradient geometries including non-linear areal-density dependence or inverted gradients will be necessary to identify the main mechanisms and geometrical parameters responsible for the striking improvement of the pinning properties of this sort of pinning landscape.

Note added in proof. While preparing this manuscript, we learned that Wang et $a l .{ }^{32}$ have investigated a particular graded array of pinning centers consisting of a conformal array of holes.

This work was partially supported by the Brazilian funding agencies FAPESP and CNPq, the Methusalem Funding of the Flemish Government, the Fund for Scientific Research-Flanders (FWO-Vlaanderen), the program for scientific cooperation F.R.S.-FNRS-CNPq, and Spanish projects CSD2007-00041 and MAT2012-35370. The work of A.V.S. was partially supported by "Mandat d'Impulsion Scientifique" of the F.R.S.-FNRS and the crédit de démarrage U.Lg.

${ }^{1}$ A. M. Campbell and J. E. Evetts, Adv. Phys. 21, 199 (1972).

${ }^{2}$ G. Blatter, M. V. Feigel'man, V. B. Geshkeinbein, A. I. Larkin, and V. M. Vinokur, Rev. Mod. Phys. 66, 1125 (1994).

${ }^{3}$ E. H. Brandt, Rep. Prog. Phys. 58, 1465 (1995).

${ }^{4}$ D. Larbalestier, A. Gurevich, D. M. Feldmann, and A. Polyanskii, Nature 414, 368 (2001).

${ }^{5}$ D. J. Morgan and J. B. Ketterson, Phys. Rev. Lett. 80, 3614 (1998).

${ }^{6}$ M. Baert, V. V. Metlushko, R. Jonckheere, V. V. Moshchalkov, and Y. Bruynseraede, Phys. Rev. Lett. 74, 3269 (1995).

${ }^{7}$ V. Vlasko-Vlasov, U. Welp, V. Metlushko, and G. W. Crabtree, Physica C 341-348, 1281 (2000).

${ }^{8}$ M. Pannetier, R. J. Wijngaarden, I. Floan, J. Rector, B. Dam, R. Griessen, P. Lahl, and R. Wördenweber, Phys. Rev. B 67, 212501 (2003).

${ }^{9}$ M. Menghini, R. J. Wijngaarden, A. V. Silhanek, S. Raedts, and V. V. Moshchalkov, Phys. Rev. B 71, 104506 (2005).

${ }^{10}$ M. Motta, F. Colauto, R. Zadorosny, T. H. Johansen, R. B. Dinner, M. G. Blamire, G. W. Ataklti, V. V. Moshchalkov, A. V. Silhanek, and W. A. Ortiz, Phys. Rev. B 84, 214529 (2011).

${ }^{11}$ R. G. Mints and A. L. Rakhmanov, Rev. Mod. Phys. 53, 551 (1981).

${ }^{12}$ D. V. Denisov, A. L. Rakhmanov, D. V. Shantsev, Y. M. Galperin, and T. H. Johansen, Phys. Rev. B 73, 014512 (2006).

${ }^{13}$ V. Misko, S. Savel'ev, and F. Nori, Phys. Rev. Lett. 95, 177007 (2005). 
${ }^{14}$ V. Misko, S. Savel'ev, and F. Nori, Phys. Rev. B 74, 024522 (2006).

${ }^{15}$ V. R. Misko, D. Bothner, M. Kemmler, R. Kleiner, D. Koelle, F. M. Petters, and F. Nori, Phys. Rev. B 82, 184512 (2010).

${ }^{16}$ M. Kemmler, C. Gürlich, A. Sterck, H. Pöhler, M. Neuhaus, M. Siegel, R. Kleiner, and D. Koelle, Phys. Rev. Lett. 97, 147003 (2006).

${ }^{17}$ A. V. Silhanek, W. Gillijns, V. V. Moshchalkov, B. Y. Zhu, J. Moonens, and L. H. A. Leunissen, Appl. Phys. Lett. 89, 152507 (2006).

${ }^{18}$ C. P. Bean, Phys. Rev. Lett. 8, 250 (1962); Rev. Mod. Phys. 36, 31 (1964).

${ }^{19}$ R. A. Richardson, O. Pla, and F. Nori, Phys. Rev. Lett. 72, 1268 (1994).

${ }^{20}$ C. Reichhardt, C. J. Olson, J. Groth, S. Field, and F. Nori, Phys. Rev. B 52, 10441 (1995).

${ }^{21}$ V. R. Misko and F. Nori, Phys. Rev. B 85, 184506 (2012).

${ }^{22}$ P. H. Kes and C. C. Tsuei, Phys. Rev. B 28, 5126 (1983).

${ }^{23}$ L. E. Helseth, R. W. Hansen, E. I. Ilyashenko, M. Baziljevich, and T. H. Johansen, Phys. Rev. B 64, 174406 (2001).

${ }^{24}$ E. Altshuler and T. H. Johansen, Rev. Mod. Phys. 76, 471 (2004).

${ }^{25}$ M. Motta, F. Colauto, W. A. Ortiz, J. I. Vestgarden, T. H. Johansen, J. Cuppens, V. V. Moshchalkov, and A. V. Silhanek, e-print arXiv:1109. 2532 (2011).
${ }^{26}$ Flux avalanches in superconducting films develop only below an upper threshold temperature, which is characteristic of each sample. For the sample named Plain in this work, this threshold temperature is $2.6 \mathrm{~K}$, as determined by magnetic measurements, a value that we cannot achieve in the cold-finger type cryostat of the magneto-optical imaging setup. We present instead an avalanche observed on a sister plain film of larger dimensions $(2.5 \mathrm{~mm} \times 2.5 \mathrm{~mm})$, at $\mathrm{T}=2.8 \mathrm{~K}$ and $\mathrm{H}=2.2 \mathrm{Oe}$.

${ }^{27}$ S. Jin, H. Mavoori, C. Bower, and R. B. van Dover, Nature 411, 563 (2001).

${ }^{28}$ M. Baziljevich, A. V. Bobyl, D. V. Shantsev, E. Altshuler, T. H. Johansen, and S. I. Lee, Physica C 369, 93 (2002).

${ }^{29}$ F. Colauto, E. Choi, J. Y. Lee, S. I. Lee, E. J. Patiño, M. G. Blamire, T. H. Johansen, and W. A. Ortiz, Appl. Phys. Lett. 96, 092512 (2010).

${ }^{30}$ F. Colauto, E. J. Patiño, M. G. Blamire, and W. A. Ortiz, Supercond. Sci. Technol. 21, 045018 (2008).

${ }^{31}$ D. Ray, C. J. Olson Reichhardt, B. Jankó, and C. Reichhardt, e-print arXiv:1210.1229v1 (2012).

${ }^{32}$ Y. L. Wang, M. L. Latimer, Z. L. Xiao, R. Divan, L. E. Ocola, G. W. Crabtree, and W. K. Kwok, private communication (2013). 